CARNETS DE Carnets de géographes

GÉOGRAPHES.

$14 \mid 2020$

Varia

\title{
Teaching Geography in English
}

Retours pédagogiques pour une hybridation des disciplines

\section{Claire Doussard}

\section{(2) OpenEdition}

Journals

Édition électronique

URL : https://journals.openedition.org/cdg/5837

DOI : $10.4000 /$ cdg. 5837

ISSN : 2107-7266

Éditeur

UMR 245 - CESSMA

Référence électronique

Claire Doussard, "Teaching Geography in English », Carnets de géographes [En ligne], 14 | 2020, mis en ligne le 07 décembre 2020, consulté le 22 mai 2021. URL : http://journals.openedition.org/cdg/5837 ; DOI : https://doi.org/10.4000/cdg.5837

Ce document a été généré automatiquement le 22 mai 2021.

\section{(c) (1)}

La revue Carnets de géographes est mise à disposition selon les termes de la Licence Creative

Commons Attribution - Pas d'Utilisation Commerciale - Pas de Modification 4.0 International. 


\title{
Teaching Geography in English
}

Retours pédagogiques pour une hybridation des disciplines

\author{
Claire Doussard
}

\section{Introduction}

1 L'enseignement de la géographie en anglais commence progressivement à faire l'objet de réflexions didactiques, notamment dans le second degré, comme en témoigne par exemple le parcours de formation proposé par le réseau Canopé (2019). Malgré la multiplication de ressources susceptibles de nourrir ces cours de géographie anglophone, notamment en ligne (la Clé des langues, site web Bitesize de la BBC, Ted Talk, etc.), et l'apparition de rares retours d'expériences (Milhaud, 2017), peu de réflexions existent encore au niveau universitaire. Celles-ci sont pourtant essentielles au renouvellement des programmes et pédagogies utilisés. De ce fait, Olivier Milhaud décrivait la portée heuristique d'un échec pédagogique concernant l'enseignement d'un cours de géographie en anglais, tout en rappelant l'importance de l'apprentissage de cette langue pour le futur chercheur géographe (Milhaud, 2005; CNFG, 2015). Cependant, tout échec est également source d'enseignements. Le présent article a donc pour objectif de contribuer à la littérature sur le sujet, et apporte un autre regard, sans doute plus optimiste, sur la réalisation de cours en géographie-aménagement anglophones.

2 Au cours des années universitaires 2017-2018 et 2018-2019, j'ai occupé un poste de contractuelle équivalent PRAG à l'Université de Cergy Pontoise, au sein de l'UFR Lettres et Sciences Humaines (LSH). Cette UFR rassemble les départements de Lettres modernes et d'Histoire-Géographie. Dans le domaine de la géographie, l'université propose une licence en géographie-aménagement, une licence professionnelle «Transport de voyageurs », et sept masters en géographie-aménagement.

3 J'ai été recrutée en tant que professeure d'anglais contractuelle, mais je suis à l'origine ingénieure-paysagiste, urbaniste, et docteure en aménagement. Ma formation d'ingénieure-paysagiste m'a permis d'enseigner les fondamentaux relatifs à l'aménagement sur le plan des techniques en lien avec les sciences naturelles. Mon 
cursus d'urbaniste aux États-Unis m'a quant à lui permis de pouvoir enseigner des notions de sciences humaines en anglais, et en général dans le cadre d'un terrain anglosaxon. À mon arrivée à l'université de Cergy Pontoise, il est apparu rapidement que mon statut principal n'était pas celui d'une urbaniste, mais celui d'une professeure de langues. Les disciplines sont alors clairement cloisonnées. Mon service établi pour la première année d'enseignement à l'UFR LSH était réparti sur les trois filières (Lettres modernes, Histoire et Géographie). Lors de ma deuxième année, j'ai cependant enseigné exclusivement en licence de géographie-aménagement, et au sein de cinq masters de géographie-aménagement de l'UFR. Je ne correspondais cependant pas au profil traditionnel du professeur d'anglais. De manière générale, ce dernier répond à trois grands types de profils. De par sa formation, le professeur d'anglais présente en général un profil de linguiste, de littéraire ou de civilisationniste britannique ou américain (Conseil National des Universités, 2019). Par conséquent, les programmes de langues pour les filières en Lettres Modernes et en Histoire sont plutôt bien établis. En revanche, les enseignants de langues sont très rarement formés à la géographie et à l'aménagement, ce qui les conduit à donner des cours naturellement orientés sur des notions de linguistique, de littérature ou de civilisation à leurs étudiants géographes. À mon arrivée dans l'établissement, il existait donc peu d'adéquation entre l'enseignement de l'anglais et celui de la géographie et de l'aménagement. Je notais également une absence de programme pédagogique construit et continu de la L1 au M2 pour la filière Géographie.

4 Aussi, mes deux années d'expérience au sein de l'UFR LSH m'ont conduite à m'interroger sur les méthodes de transmission d'un savoir pour un public de géographes, principalement en langue étrangère. Mes questionnements s'articulaient autour des deux thématiques suivantes :

- Comment allier enseignement de géographie-aménagement et enseignement linguistique?

- Comment adapter mes cours à une classe d'étudiants au niveau hétérogène, tant en géographie-aménagement qu'en maitrise de l'anglais?

5 Cet article se concentre sur ma deuxième année d'enseignement (2018-2019), où j'ai enseigné exclusivement en géographie. Il s'intéresse à la construction d'un programme pédagogique en géographie-aménagement anglophone, de la L1 au M2, ainsi qu'au développement d'une pédagogie d'enseignement innovante. Enfin, il examine l'évaluation du programme et de la pédagogie établis, au travers de retours d'étudiants à l'issue des cours par l'intermédiaire de la réalisation d'un google form anonymisé au niveau Master.

\section{Programme de géographie-aménagement anglophone}

L'élaboration d'un programme pédagogique s'est révélé être un défi, par la réflexion que celui-ci a nécessité à la fois en géographie et en aménagement, mais aussi au niveau linguistique.

7 En premier lieu, il était nécessaire de distinguer les niveaux d'études, non seulement entre la licence et le master, mais aussi entre les filières. En effet, en licence, les règles pédagogiques relatives à l'enseignement de la grammaire anglaise et à l'évaluation des connaissances sont assez similaires pour les trois filières, que ce soit en lettres modernes, en histoire ou en géographie-aménagement. L'objectif est alors d'éviter les 
disparités d'évaluation entre les groupes, et faciliter l'intégration de nouveaux enseignants vacataires ou contractuels qui ont alors des directives pédagogiques précises. En master, il existe une plus grande flexibilité et marge de manœuvre dans le développement des enseignements et des modalités d'évaluation. L'enseignant est responsable du programme pédagogique, suite à des discussions avec les différents directeurs de master.

8 En deuxième lieu, l'enseignement linguistique oriente en général le contexte régional dans lequel s'inscrit le cours. L'alternance entre des cours plutôt orientés civilisation britannique ou civilisation américaine (avec quelques incursions ailleurs dans le monde) est favorisée. Cette alternance contribue à l'élaboration de cours en géographie régionale, et permet également de transmettre des savoirs théoriques appliqués à différents territoires et cultures anglo-saxons.

Un autre paramètre guidant la réalisation d'un programme en géographieaménagement anglophone est la correspondance avec les maquettes pédagogiques $\mathrm{du}$ département. En effet, afin de faciliter l'apprentissage linguistique, j'ai décidé de revoir les notions de géographie et d'aménagement enseignées par mes collègues géographes en français, que j'applique alors au sein d'autres territoires anglophones. L'objectif est alors de permettre aux étudiants de revoir et d'approfondir des notions normalement déjà acquises, de les manipuler au prisme du territoire britannique et américain, tout en apprenant du vocabulaire de spécialité en anglais.

Enfin, un dernier paramètre à prendre en compte est le niveau de langue des étudiants établi par le Cadre Européen Commun de Référence pour les Langues (CECRL). Ce cadre est un document datant de 2001 qui définit pour toute langue étrangère un niveau de maîtrise et de savoir-faire linguistique (Conseil de l'Europe, 2019). Il existe six niveaux de langues, A1, A2, B1, B2, C1, C2, le niveau A1 étant qualifié d'« introductif », le niveau C2 quant à lui atteste d'une maîtrise avancée de la langue. En raison d'un grand nombre d'étudiants entrant en première année de licence (L1), l'UFR établit trois groupes de travaux dirigés (TD) en fonction de leur niveau linguistique. Cependant, en L2, L3 puis en master, un plus petit nombre d'étudiants ne permet d'établir qu'une seule classe en anglais. L'hétérogénéité des niveaux de langues des étudiants est alors un défi à surmonter lorsqu'il s'agit d'enseigner des compétences linguistiques associées à des notions géographiques de plus en plus complexes.

11 En raison de tous ces paramètres, j'ai pu établir le programme suivant pour l'année 2018-2019.

Tableau 1. Programme des cours de géographie-aménagement anglophone enseignés pour l'année universitaire 2018-2019

\begin{tabular}{|l|l|l|l|}
\hline $\begin{array}{l}\text { Niveau } \\
\text { d'étude et } \\
\text { spécialité }\end{array}$ & Titre du cours & Notions géographiques & Notions linguistiques \\
\hline L1S1 & $\begin{array}{l}\text { Introduction to } \\
\text { anglophone territories 1 }\end{array}$ & $\begin{array}{l}\text { Bases géographiques des } \\
\text { différents territoires } \\
\text { d'étude : Royaume-Uni, } \\
\text { Irlande, Océanie }\end{array}$ & $\begin{array}{l}\text { Vocabulaire géographique de } \\
\text { base 1 } \\
\text { Révisions de grammaire au } \\
\text { programme établi par le } \\
\text { département }\end{array}$ \\
\hline
\end{tabular}




\begin{tabular}{|c|c|c|c|}
\hline L1S2 & $\begin{array}{l}\text { Introduction to } \\
\text { anglophone territories } 2\end{array}$ & $\begin{array}{l}\text { Bases géographiques des } \\
\text { différents territoires } \\
\text { d'étude : } \quad \text { Etats-Unis, } \\
\text { Canada, Caraibes }\end{array}$ & $\begin{array}{l}\text { Vocabulaire géographique de } \\
\text { base } 2 \\
\text { Révisions de grammaire au } \\
\text { programme établi par le } \\
\text { département }\end{array}$ \\
\hline L2S3 & $\begin{array}{l}\text { Territorial politics in a } \\
\text { transitional society: the } \\
\text { American case }\end{array}$ & $\begin{array}{l}\text { Géographie politique et } \\
\text { sociale des Etats-Unis }\end{array}$ & $\begin{array}{l}\text { Vocabulaire de spécialité en } \\
\text { lien avec la géographie sociale } \\
\text { et politique } \\
\text { Révisions de grammaire au } \\
\text { programme établi par le } \\
\text { département }\end{array}$ \\
\hline L3S4 & $\begin{array}{l}\text { British geomorphology } \\
\text { and landscapes: Origins } \\
\text { and evolution }\end{array}$ & $\begin{array}{lll}\text { Géographie physique et } & \text { é } \\
\text { géologie } & \text { des îles } \\
\text { britanniques } & & \end{array}$ & $\begin{array}{l}\text { Vocabulaire de spécialité en } \\
\text { lien avec la géographie } \\
\text { physique et la géologie } \\
\text { Révisions de grammaire au } \\
\text { programme établi par le } \\
\text { département }\end{array}$ \\
\hline L3S5 & $\begin{array}{lr}\text { A short } & \text { history of } \\
\text { American } & \text { urban } \\
\text { geographies, } & \text { planning } \\
\text { and design } & \end{array}$ & $\begin{array}{l}\text { Histoire de l'urbanisme et } \\
\text { aménagement aux Etats- } \\
\text { Unis, XIX ème et XX ème } \\
\text { siècles }\end{array}$ & $\begin{array}{l}\text { Vocabulaire de spécialité en } \\
\text { lien avec l'urbanisme et } \\
\text { l'aménagement du territoire. } \\
\text { Révisions de grammaire } \\
\text { ponctuelles en fonction de la } \\
\text { demande des étudiants }\end{array}$ \\
\hline L3S6 & $\begin{array}{l}\text { Smart, sustainable, } \\
\text { resilient : future British } \\
\text { territories }\end{array}$ & $\begin{array}{l}\text { Aménagement et } \\
\text { prospective territoriale au } \\
\text { Royaume-Uni, XXI }{ }^{\text {ème }} \text { siècle }\end{array}$ & $\begin{array}{l}\text { Vocabulaire de spécialité en } \\
\text { lien avec le développement } \\
\text { durable, le numérique et la } \\
\text { résilience des territoires } \\
\text { Préparation au TOEIC }{ }^{1} \text { grâce au } \\
\text { logiciel Global Exam }\end{array}$ \\
\hline $\begin{array}{l}\text { M1 TTE- } \\
\text { EDRTP }^{2}\end{array}$ & $\begin{array}{l}\text { Developing public } \\
\text { transportation } \\
\text { in the United States : } \\
\text { Atlanta }\end{array}$ & $\begin{array}{l}\text { Analyse géographique et } \\
\text { territoriale du transport } \\
\text { public aux Etats-Unis. } \\
\text { Atelier de projet sur } \\
\text { Atlanta }\end{array}$ & $\begin{array}{l}\text { Vocabulaire de spécialité en } \\
\text { lien avec les transports publics } \\
\text { Révisions de grammaire grâce } \\
\text { au logiciel Gymglish adaptées } \\
\text { en fonction de l'étudiant }\end{array}$ \\
\hline $\begin{array}{l}\text { M2 TTE- } \\
\text { EDRTP }\end{array}$ & $\begin{array}{l}\text { Understanding public } \\
\text { transportation } \\
\text { management: London }\end{array}$ & $\begin{array}{l}\text { Gestion de la demande et } \\
\text { de l'offre de transport } \\
\text { public en Grande Bretagne. } \\
\text { Atelier de projet sur } \\
\text { Londres }\end{array}$ & $\begin{array}{l}\text { Vocabulaire de spécialité en } \\
\text { lien avec la gestion de l'offre } \\
\text { de transport de voyageurs } \\
\text { Révisions de grammaire grâce } \\
\text { au logiciel Gymglish adaptées } \\
\text { en fonction de l'étudiant. } \\
\text { Préparation au TOEIC }\end{array}$ \\
\hline
\end{tabular}




\begin{tabular}{|c|c|c|c|}
\hline $\mathrm{M} 1 \mathrm{ML}^{3}$ & $\begin{array}{l}\text { Management and the } \\
\text { future of urban } \\
\text { logistics:Chicago }\end{array}$ & $\begin{array}{l}\text { Analyse de circuits } \\
\text { logistiques en lien avec le } \\
\text { transport de fret, et } \\
\text { prospective urbaine. } \\
\text { Atelier de projet sur } \\
\text { Chicago }\end{array}$ & $\begin{array}{l}\text { Vocabulaire de spécialité en } \\
\text { lien avec la gestion de l'offre } \\
\text { de transport de marchandises } \\
\text { Révisions de grammaire grâce } \\
\text { au logiciel Gymglish adaptées } \\
\text { en fonction de l'étudiant }\end{array}$ \\
\hline M1 GAEUR $^{4}$ & $\begin{array}{l}\text { Assessing climate } \\
\text { vulnerability and } \\
\text { developing resilience for } \\
\text { NOLA }\end{array}$ & $\begin{array}{l}\text { Diagnostic territorial en } \\
\text { lien avec le changement } \\
\text { climatique. } \\
\text { Atelier de projet sur la } \\
\text { Nouvelle-Orléans }\end{array}$ & $\begin{array}{l}\text { Vocabulaire de spécialité en } \\
\text { lien avec la géomatique et } \\
\text { l'analyse des risques } \\
\text { climatiques } \\
\text { Révisions de grammaire grâce } \\
\text { au logiciel English AZ adaptées } \\
\text { en fonction de l'étudiant }\end{array}$ \\
\hline M2 GAEUR & $\begin{array}{l}\text { Understanding, } \\
\text { assessing, and } \\
\text { responding to terrorism } \\
\text { in London }\end{array}$ & $\begin{array}{l}\text { Analyse de risques en lien } \\
\text { avec le terrorisme. } \\
\text { Atelier de projet sur } \\
\text { Londres }\end{array}$ & $\begin{array}{l}\text { Vocabulaire de spécialité en } \\
\text { lien avec la géomatique et } \\
\text { l'analyse des risques en lien } \\
\text { avec le terrorisme } \\
\text { Préparation au TOEIC grâce au } \\
\text { logiciel Global Exam }\end{array}$ \\
\hline M1 DCVP 5 & $\begin{array}{l}\text { Park preservation in the } \\
\text { United States }\end{array}$ & $\begin{array}{l}\text { Développement d'une offre } \\
\text { culturelle en lien avec les } \\
\text { parcs nationaux } \\
\text { américains. } \\
\text { Atelier de projet sur Anza } \\
\text { Borrego Desert Park, } \\
\text { Californie }\end{array}$ & $\begin{array}{l}\text { Vocabulaire de spécialité en } \\
\text { lien avec le domaine de la } \\
\text { culture et la préservation du } \\
\text { patrimoine naturel } \\
\text { Révisions de grammaire grâce } \\
\text { au logiciel English } A Z \text { adaptées } \\
\text { en fonction de l'étudiant }\end{array}$ \\
\hline M2 DCVP & $\begin{array}{l}\text { Heritage preservation in } \\
\text { the UK: Kew Gardens }\end{array}$ & $\begin{array}{l}\text { Développement d'une offre } \\
\text { culturelle en lien avec le } \\
\text { patrimoine britannique. } \\
\text { Atelier de projet sur les } \\
\text { jardins botaniques royaux } \\
\text { de Kew, Londres }\end{array}$ & $\begin{array}{l}\text { Vocabulaire de spécialité en } \\
\text { lien avec le domaine de la } \\
\text { culture et la préservation du } \\
\text { patrimoine construit } \\
\text { Préparation au TOEIC grâce au } \\
\text { logiciel Global Exam }\end{array}$ \\
\hline
\end{tabular}

12 Comme le démontre le tableau 1, le programme s'appuie sur une diversification des thèmes et des territoires d'études, ainsi que sur l'adéquation entre niveaux d'études, filières, et spécialités au niveau master.

13 Cependant, l'enseignement de ce programme suppose la mise en place d'une pédagogie qui tienne compte non seulement des connaissances à transmettre en géographieaménagement mais aussi des savoirs et compétences linguistiques que les étudiants devront acquérir et entretenir. 


\section{Pédagogie et expérimentations}

14 Comme pour le programme, le développement d'une pédagogie doit s'adapter à la fois aux niveaux d'études, à la demande des collègues linguistes et géographes, et aux étudiants.

\section{En licence : le maintien d'une approche traditionnelle d'apprentissage en langue et en géographie-aménagement}

15 En licence, la pédagogie est essentiellement discutée et négociée avec l'ensemble des collègues de l'équipe de langues qui interviennent au sein des trois filières. Celle-ci est relativement traditionnelle. Elle s'appuie sur l'établissement de brochures et la mobilisation de différents médias, audio ou vidéo, en lien avec une thématique développée au cours du semestre. L'objectif est de permettre aux étudiants de mobiliser les quatre compétences langagières (lire, écrire, parler, écouter) et de les développer au cours du semestre. Le niveau B2 du CECRL est attendu à l'issue de la L3.

Cependant, chaque collègue dispose d'une certaine indépendance quant au choix des documents étudiés et quant au déroulement des TD qui durent en général deux heures. Le déroulement de mes cours s'appuie sur l'enchaînement de plusieurs séquences d'activités courtes pour minimiser la charge cognitive et mobiliser l'attention des étudiants. En effet, la charge cognitive est l'effort mental dont l'étudiant a besoin pour réaliser une tâche ou résoudre un problème dans un contexte donné (Sweller, 1988; Shraw et al., 2006). Elle est liée à sa mémoire de travail, c'est-à-dire la capacité de l'étudiant à mémoriser et manipuler les informations, et notamment au maintien de son attention. Afin de favoriser l'attention des étudiants, je partage la séance en séquences de 15 ou 30 minutes, en alternant le type d'activité. Lors des cours je fais également attention à mobiliser différents types de mémoires (visuelle, auditive) en utilisant des techniques de gestuelle et de répétition.

17 Aussi, la première heure est dédiée à l'étude d'une brochure distribuée aux étudiants en début d'année. Chaque cours commence par la lecture d'un texte thématique par les étudiants, et la correction de l'accent de ces derniers pendant 15 minutes. Le cours se poursuit par l'étude du vocabulaire de spécialité pendant 15 minutes, et la compréhension du texte et de différents documents graphiques et médias (cartes géographiques, infographies, photos d'archives, vidéo Youtube et TED talk, etc.) pendant 30 minutes. Je pose généralement des questions sur les documents et oriente la discussion pour favoriser l'expression orale des étudiants. La deuxième heure est dédiée aux révisions de grammaire pendant 30 minutes, la dernière demi-heure étant consacrée à différentes activités permettant une participation orale accrue des étudiants. Celle-ci s'oriente sur la présentation orale de travaux demandés aux étudiants, ou sur la mise en place d'un débat à partir d'une controverse en lien avec la thématique du cours.

Ces deux heures de cours permettent donc de mobiliser les quatre compétences langagières à partir de différents documents en lien avec la géographie et l'aménagement. Cependant, cette approche demeure relativement traditionnelle en raison du cadre établi par l'équipe enseignante en langues afin d'établir une formation homogène de qualité. 


\section{En master : un renouvellement des pratiques pédagogiques}

19 Au niveau master, les enseignants sont plus libres dans le développement d'une pédagogie qui leur est propre, puisqu'aucune directive de l'équipe des langues n'influence le déroulement des cours. Comme en licence, je m'appuyais sur une brochure et plusieurs types de médias pour l'apprentissage de différentes notions théoriques en lien avec la spécialité du master. Cependant, l'organisation du cours s'articule autour de la mobilisation de ces notions par la réalisation d'un atelier de projets en petits groupes de trois à cinq étudiants maximum. L'organisation des activités est donc plus fluide qu'en licence, car la durée des cours (2 heures, 3 heures ou 4 heures) varie en fonction des masters. Cette flexibilité en termes de contenu, d'organisation et d'amplitude horaire, m'a donc permis de négocier avec les différents directeurs des masters au sein desquels j'enseignais en mettant en place différentes expérimentations pédagogiques. Celles-ci s'organisent autour de deux grands points.

Le premier concerne l'apport de nouveaux outils numériques linguistiques au sein d'une formation en géographie-aménagement. Il convient en effet d'admettre que les étudiants d'une classe de master présenteront souvent des différences de niveau linguistique importantes, qui ne pourront être résolues avec vingt heures TD d'enseignement d'anglais par semestre. Un enseignant ne peut adapter son cours disciplinaire en fonction du niveau linguistique de chaque étudiant. Ces nouveaux outils permettent alors une formation linguistique non seulement personnalisée en fonction du niveau des étudiants, mais aussi en continu en dehors de l'université. En classe, je pouvais me concentrer sur mon enseignement en géographie-aménagement. À distance, je contrôlais l'évolution de leur niveau linguistique grâce à une plateforme administrateur web.

21 Trois plateformes numériques d'apprentissage ont été expérimentées durant l'année 2018-2019 en fonction des classes de master: Gymglish, EnglishAZ et Global exam. Gymglish s'est avérée la meilleure plateforme d'après les retours d'étudiants et d'enseignants. Le temps d'apprentissage linguistique des étudiants était a minima doublé, mais certains étudiants motivés ont ainsi triplé leur temps d'apprentissage pour atteindre près de soixante heures d'enseignement annualisées, à hauteur de quinze à vingt minutes par jour de pratique de l'anglais.

22 Cependant, l'apport du numérique seul ne peut garantir un bon enseignement disciplinaire. Celui-ci se combine à d'autres approches d'enseignement, qui favorisent notamment les logiques cognitives, de jeux et d'interactions, plus particulièrement lors de la mise en place d'ateliers de projet. Proposer de nouveaux savoirs et méthodes pédagogiques inhabituelles pour les étudiants ne se fait pas sans résistance. Cette notion de résistance au changement en sciences cognitives est primordiale dans le sens où elle a un impact déterminant sur l'apprentissage (Dutke et al., 2017; Silvia, 2005). Aussi, j'ai mis en place des stratégies ludiques afin que les étudiants soient ouverts à ces nouveaux apprentissages, les acceptent et se les approprient. Pour ce faire, j'ai favorisé des interactions sociales basées sur des stratégies de compétition/coopération intra- et inter-groupes, comme l'a défini Silvia (2005) dans ses travaux. 
Exemple de mise en place d'un atelier de projet sur la valorisation d'un site patrimonial britannique, les jardins botaniques royaux de Kew, en Master 2 Développement Culturel et Valorisation des Patrimoines

Le cours d'atelier commence par un test rapide pour déterminer le niveau des élèves, et constituer cinq groupes de travail de trois à cinq étudiants, de niveaux hétérogènes, afin de favoriser les dynamiques de soutien. Chaque groupe doit également se créer une identité propre, avec un logo, une devise, et un rôle pour lequel ils doivent assumer les codes tout au long du semestre. Cela peut être le cas d'un groupe qui souhaite devenir une équipe d'assistance à maîtrise d'ouvrage ou de conseil pour un projet d'aménagement donné. Tous les groupes sont encouragés à interagir avec des professionnels extérieurs à l'université en envoyant des mails de demande d'informations aux jardins botaniques royaux par exemple.

Dans le cadre de cet atelier, huit séances sont organisées pour réaliser progressivement les différentes parties d'un rapport écrit et d'une présentation évaluée à la fin du semestre devant un professionnel anglophone extérieur invité dans le cadre d'un « Crit » (l'équivalent d'un jury). Chaque séance s'articule autour de l'analyse d'un corpus de ressources anglophones (textes, images et vidéos) réunies au sein d'une brochure, susceptibles d'informer le développement du projet tout en permettant de développer les compétences linguistiques des étudiants. Le planning des séances spécifiquement orientées sur l'atelier (d'autres sont réservées à la pratique du TOEIC au cours du semestre) est présenté dans le tableau 2.

Chaque groupe développe alors un projet qui lui est propre au cours du semestre. La figure 1 présente des extraits du rendu du groupe " La French Touch », ayant travaillé sur la mise en place de stations de réalité virtuelle au sein des jardins.

Enfin, chaque équipe est en compétition avec les autres groupes de la classe lors des phases de tests (de compréhension, de vocabulaire) sous la forme de quizz hebdomadaires pour lesquels ils peuvent gagner des points, ou en perdre. L'équipe qui a gagné le plus de points à la fin de l'année ou du semestre a le droit à une récompense : une valorisation de la note de tous les membres de l'équipe. Ma notation prend non seulement en compte l'évaluation du contenu disciplinaire, mais aussi la régularité de l'apprentissage et la coopération entre membres d'un même groupe.

Tableau 2. Programme des séances d'atelier

\begin{tabular}{|l|l|}
\hline Séance 1 & $\begin{array}{l}\text { Constitution des équipes } \\
\text { Brainstorming sur des thématiques relatives à la préservation du patrimoine }\end{array}$ \\
\hline Séance 2 & Premières recherches concernant les jardins botaniques royaux de Kew (site UNESCO) \\
\hline Séance 3 & Diagnostic territorial et définition des enjeux et besoins \\
\hline Séance 4 & Premières idées d'aménagement et benchmark \\
\hline
\end{tabular}




\begin{tabular}{|l|l|}
\hline Séance 5 & $\begin{array}{l}\text { Présentations de mi-semestre } \\
\text { Feedback et nouveau brainstorming à partir des idées débattues lors des présentations }\end{array}$ \\
\hline Séance 6 & Développement du projet et mise en place de stratégies d'aménagement \\
\hline Séance 7 & Gouvernance et partenariats \\
\hline Séance 8 & Crit : Présentations finales \\
\hline
\end{tabular}

Figure 1. Exemple de rendu d'un groupe d'étudiants

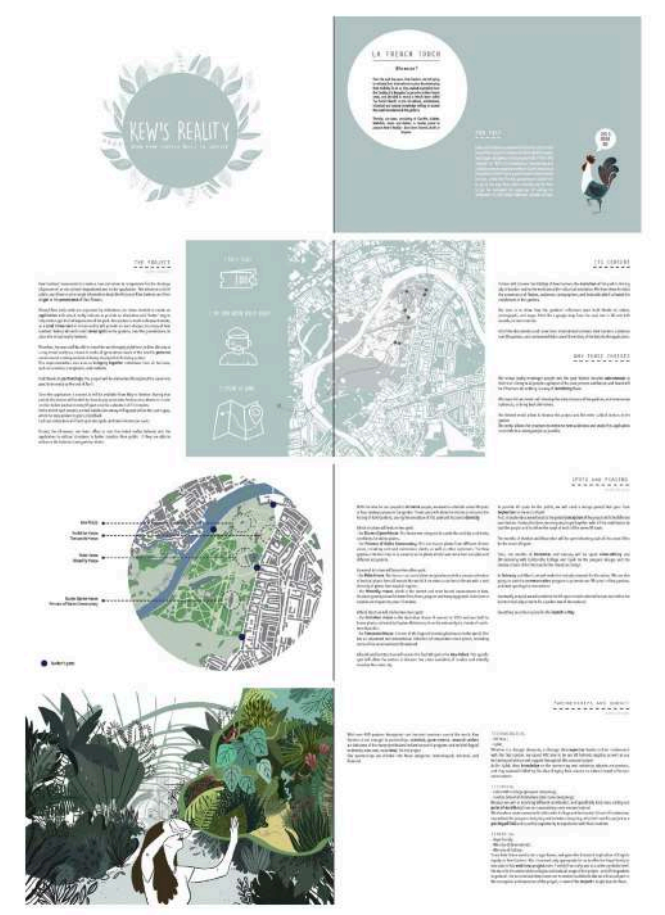

Source : groupe La French Touch (Anzanello Camille, Adrian Buisson, Juliette Epain, Mathilde Lull, Jason Ribot-Carlini), 2019

\section{Évaluation des expérimentations pédagogiques par les étudiants de master}

À l'issue d'une année d'expérimentation pédagogique, j'ai proposé un questionnaire d'évaluation de mes cours à l'ensemble de mes étudiants de master. Quelques éléments relatifs à la répartition des étudiants et des réponses obtenues sont présentés dans le tableau 3. 
Tableau 3. Répartition des effectifs en fonction du taux de réponse au questionnaire et du statut des étudiants

\begin{tabular}{|l|l|l|l|l|}
\hline Master & $\begin{array}{l}\text { Effectif } \\
\text { total }\end{array}$ & $\begin{array}{l}\% \\
\text { répondants }\end{array}$ & $\begin{array}{l}\% \text { de répondants } \\
\text { femme }\end{array}$ & $\begin{array}{l}\% \text { d'étudiants dont la formation est } \\
\text { financée }{ }^{6}\end{array}$ \\
\hline TTE & 36 & 69,4 & 48 & 88,9 \\
\hline EDRTP & 41 & 87,8 & 41,7 & 97,6 \\
\hline DCVP & 39 & 64,1 & 88 & 69,2 \\
\hline GAEUR & 44 & 63,6 & 28,6 & 68,2 \\
\hline ML & 26 & 69,2 & 38,9 & 65,4 \\
\hline
\end{tabular}

Le questionnaire, anonymisé, et réalisé grâce à l'application google form était construit en 4 parties, quel que soit le master :

- Partie 1 : évaluation de l'enseignement de la langue en présentiel (7 questions) ;

- Partie 2: évaluation de l'enseignement de la langue à partir de l'utilisation de plateformes numériques (5 questions);

- Partie 3 : évaluation de l'enseignement en géographie-aménagement (5 questions);

- Partie 4 : évaluation sur le déroulement du cours et sur la pédagogie (9 questions).

Les questions sont adaptées en fonction des masters et des plateformes numériques utilisées, mais cherchent à chaque fois à évaluer les mêmes compétences et fondements pédagogiques. Le questionnaire s'appuie sur des questions présentant différents degrés d'ouverture pour permettre à la fois d'obtenir des résultats quantitatifs et qualitatifs pertinents. Les questions fermées demandent par exemple à l'étudiant d'évaluer sur une échelle de 1 à 5 l'amélioration perçue de certaines compétences. Les questions les plus ouvertes sont associées à des espaces de commentaires libres des étudiants sur des aspects précis de la formation, par exemple leur avis sur la plateforme numérique, ou les aspects du cours à améliorer. Plusieurs grandes tendances ressortent des retours des étudiants, sur l'enseignement linguistique, en géographie-aménagement et sur la pédagogie mise en place.

En premier lieu, les étudiants perçoivent une claire progression de leur connaissance du vocabulaire de spécialité. Celle-ci est évaluée à 3,74/5, sur une échelle allant de 1 (je n'ai pas l'impression d'avoir appris du vocabulaire) à 5 (j'ai tout à fait l'impression d'avoir appris du vocabulaire). Ceci est dû à l'étude et à la production de textes et de documents opérationnels de la profession en lien avec leur spécialité. Les étudiants perçoivent une moindre progression en grammaire, évaluée à 2,75/5 sur une échelle allant de 1 (je n'ai pas l'impression d'avoir progressé en grammaire) à 5 (j'ai tout à fait l'impression d'avoir progressé en grammaire). Toutefois, les logiciels, notamment Gymglish, viennent contredire l'auto-évaluation des étudiants, avec une progression de 0,34 points de moyenne sur une échelle allant de 1 (niveau A1 du CECRL) à 5,5 (niveau C2 du CECRL). Ce résultat paradoxal, qui confronte l'évaluation à priori objective d'un logiciel sur la base d'indicateurs de performance linguistique, avec la perception des étudiants vis-à-vis de leur progression personnelle, pose la question de la pertinence de l'hybridation entre enseignement en présentiel et à distance, et de la nécessité du 
professeur en tant qu'élément essentiel de l'apprentissage. Il faut également faire la différence entre les diverses plateformes numériques aujourd'hui proposées sur le marché, qui ne sont pas toutes optimales pour l'enseignement de la langue auprès d'étudiants de master. Dans le cas qui nous intéresse, la plateforme Global Exam est la plus appréciée des étudiants, dont la totalité recommande de poursuivre la formation grâce à ce logiciel. Cependant, ce dernier demeure limité à la préparation au TOEIC, et ne permet pas un apprentissage holistique de la langue. La plateforme Gymglish est également appréciée des étudiants : $87 \%$ recommandent de poursuivre la formation avec ce logiciel. Cette plateforme permet en outre de pratiquer l'ensemble des compétences linguistiques de manière transverse. Elle est également la plus ergonomique en terme de pilotage par le professeur. Ce logiciel est néanmoins le plus coûteux des trois mis en place durant l'année. Enfin, le logiciel English $A Z$, qui est quant à lui gratuit, est le moins plébiscité des étudiants, puisque seulement $55 \%$ d'entre eux le recommande dans la poursuite de la formation. Il est à noter également qu'en moyenne, seulement $35 \%$ des étudiants utilisaient régulièrement ce dernier logiciel. Malgré ces constats, l'ensemble des étudiants note une amélioration de leur niveau d'anglais au cours de l'année, avec une moyenne de 3,56/5, sur une échelle allant de 1 (je n'ai pas l'impression d'avoir progressé en anglais cette année) à 5 (j'ai l'impression d'avoir progressé cette année). Cette impression est confirmée par l'ensemble des résultats enregistrés par les plateformes numériques et par la progression des scores de TOEIC au cours de l'année pour les étudiants de $2^{\text {ème }}$ année des quatre masters au sein desquels j'ai enseigné. Cette progression est alors en moyenne de 21 points pour le TOEIC, même si l'ensemble des étudiants peine à atteindre le niveau B2 également requis en fin de Master.

Les étudiants notent également une progression dans l'apprentissage des notions en géographie-aménagement, avec une moyenne de 3,77/5 sur l'ensemble des masters. L'application de certaines notions théoriques à des territoires anglophones sous le format d'atelier est également appréciée des étudiants, tout en représentant parfois un défi pour ceux ayant le plus de difficultés à s'exprimer en anglais.

« Le travail sur une vraie ville, aux modes de transports variés qui plus est, est très intéressant. Donc continuez ainsi à travailler sur des vrais dossiers comme nous avons pu le faire, avec de la profondeur de réflexion, une étude des enjeux, et une production finale. C'est bien plus intéressant que de dire pour une énième fois que Brian est dans cette maudite cuisine!»

«Le projet a été pour moi extrêmement compliqué à comprendre. Beaucoup d'importance lui est accordé, au détriment de l'anglais, qui incarne finalement un obstacle à la conduite de celui-ci (difficultés de compréhension et, surtout, difficultés de communication avec les autres). »

Ces expériences étudiantes contrastées traduisent la difficulté de développer un cours anglophone pour une classe présentant de fortes disparités de niveau de langues. Les innovations pédagogiques ludiques mises en place afin de « décoincer » les étudiants et favoriser les interactions en classes ont été plus ou moins bien évaluées en fonction des masters et des étudiants. Alors que dans certaines classes, la mise en place d'une compétition amicale a été très appréciée et a permis une réelle émulation, dans d'autres, elle s'est avérée être un facteur d'isolement et d'angoisse parmi les étudiants les plus faibles.

«Le jeu des équipes en début de cours, est une très bonne idée qui pousse tout le monde à participer activement de manière ludique. » 
À l'opposé :

«Je n'ai pas apprécié la compétition de vocabulaire qui n'est pas indispensable

selon moi.»

Certaines classes sont donc de nature plus coopérative ou compétitive. Cependant, il est difficile à ce stade d'établir un lien entre ces différences d'opinion constatées et le genre, le taux d'étudiants dont la formation est complétement financée, ou encore la «culture » de chaque master. Bien que le profil social des étudiants eût été un atout dans la compréhension du rapport que ces derniers entretiennent à la compétition ludique, je ne dispose cependant pas à ce jour des informations permettant d'établir de telles corrélations.

31 La constitution de groupes de niveau hétérogène ne tenant pas compte des amitiés au sein de la classe a également eu des effets parfois pervers sur la cohésion de certains groupes et rendus.

«Pouvoir choisir les membres du groupe car seulement se baser sur le niveau individuel n'est pas vraiment pratique. La cohésion de groupe n'a pas franchement été bonne pour nous, ni même pratique pour mener le projet à bien. »

Cependant, les cas de mésentente au sein des groupes étaient relativement marginaux ; c'est pour cela que j'ai rendu la formation des groupes plus souple l'année suivante.

\section{Conclusion}

33 À l'issue de cette année d'expérimentation, l'heure est au bilan. Contrairement à Olivier Milhaud (2017), je ne pense pas que cette expérience soit perçue comme un échec ou comme une réussite, par les étudiants comme par l'enseignant. Cette expérience indique au contraire que tout établissement de programme et expérimentation pédagogique est perfectible. L'enseignement de la géographie et de l'aménagement en langue anglaise est certes un défi, mais il m’a poussée à me poser de véritables questions pédagogiques et à rester humble face au fait qu'on ne peut jamais satisfaire l'ensemble des étudiants. Est-il alors possible d'adapter sa pédagogie et le contenu des cours à chaque master ? À chaque étudiant? N'est-ce pas trop attendre d'une formation souvent courte (entre vingt heures et cinquante-trois heures TD annualisées) pour permettre une réelle progression des étudiants en langue?

34 La réalisation de cours de géographie-aménagement anglophones pose également la question du recrutement d'enseignants compétents à la fois en géographieaménagement et en langues. En effet, l'enseignant géographe linguiste représente un profil relativement rare dans l'enseignement supérieur. Alors que plus de $20 \%$ des enseignants de langues dans le supérieur en 2014 étaient considérés comme non permanents (Ministère de l'enseignement supérieur et de la recherche, 2020), ceci questionne la capacité des universités à établir une continuité pédagogique de qualité en recrutant du personnel compétent, et ce, de manière plus pérenne.

Enfin, à titre personnel, ma réelle interrogation concerne celle de mon positionnement en tant que professionnelle de l'enseignement. Suis-je une enseignante d'anglais? De géographie? Un hybride qui ne rentre dans aucune case administrative ? Cela traduit à mon sens un manque de porosité entre des disciplines qui gagneraient pourtant manifestement à favoriser et développer les échanges entre elles. L'hybridation pourrait alors constituer une réponse à un enseignement qui se cherche encore. 


\section{BIBLIOGRAPHIE}

Canopé (2019), Enseignement en DNL : histoire-géographie. [En ligne : https://www.reseau-canope.fr/ notice/enseignement-en-dnl-histoire-geographie.html, consulté le 2 septembre 2019

CNFG (2014), Langue, production et diffusion scientifiques dans la mondialisation: Une question pour les géographies et les géographes, Forum Francophone, Paris. [En ligne : http://cnfg.fr/images/stories/ docs_CNFG/2015/Rapport-CNFG-langues-de-la-diffusion-scientifique_geographie.pdf, consulté le 2 septembre 2019]

Conseil de l'Europe (2019), Cadre européen commun de référence pour les langues. Apprendre, enseigner, évaluer. [En ligne, https://www.coe.int/fr/web/common-european-framework-referencelanguages, consulté le 2 septembre 2019]

Conseil National des Université (2019), Section 11 : Etudes anglophones. [En ligne : https:// www.conseil-national-des-universites.fr/cnu/\#/entite/entiteName/CNU/idChild/32, consulté le 2 septembre 2019]

Dukte S., Bakker H., Papageorgi J., Taylor J., Dukte S., et al. (2017), « Evidence-based TeachingExamples from Learning and Teaching Psychology », Psychology Learning and Teaching, no. 16, pp. 175-178.

Milhaud O. (2005), « Les géographes parlent-ils tous du même monde ? Les réseaux intellectuels : Hégémonie anglo-américaine et vision du monde », Actes du Festival International de Géographie de Saint-Dié.

Milhaud O. (2017), « Teaching geography in English : Let's do it ? Portée heuristique d'un échec pédagogique », Carnets de Géographes, no. 10. [En ligne : http://journals.openedition.org/cdg/ 1173]

Ministère de l'Enseignement Supérieur et de la Recherche (2020), Les enseignants non permanents affectés dans l'enseignement supérieur - Année 2018-2019, Note de la DGRG. [En ligne : https:// cache.media.enseignementsup-recherche.gouv.fr/file/statistiques/38/6/ Note_DGRH_n4_Mai_2020_-_Enseignants_non_permanents_2018-2019_1299386.pdf]

Schraw G., Kent C., Hartley K. (2006), « Promoting self-regulation in science education: Metacognition as part of a broader perspective on learning ", Research in Science Education, no. 36, vol. 1-2, pp. 111-139.

Silvia P. (2005), « Deflecting reactance : The role of similarity in increasing compliance and reducing resistance », Basic and Applied Social Psychology, no. 27, vol. 3, pp. 277-284.

Sweller J. (1988), « Cognitive load during problem solving : Effects on learning », Cognitive Sciences, no. 12, vol. 2, pp. 257-285.

\section{NOTES}

1. Le Test of English for International Communication (TOEIC) est une certification standardisée créée et gérée par l'organisme Education Testing Service (ETS) aux États-Unis, qui permet d'évaluer le niveau d'anglais des locuteurs non anglophones à l'oral et à l'écrit.

2. TTE : Transport, Territoire, Environnement. EDRTP : Exploitation et Développement des Réseaux de Transports Publics 
3. ML : Management Logistique.

4. GAEUR : Géomatique Appliquée aux Etudes Urbaines et aux Risques.

5. DCVP : Développement Culturel et Valorisation des Patrimoines.

6. Les étudiants dont la formation est financée sont soit en apprentissage, soit en contrat pro, ou en formation continue. Les étudiants étant en formation initiale sont quant à eux autofinancés.

\section{RÉSUMÉS}

Cet article interroge la mise en place d'une pédagogie et d'un programme de géographieaménagement anglophone spécifiques aux niveaux Licence et Master à l'Université de CergyPontoise. Il démontre qu'une hybridation entre les disciplines, en géographie-aménagement et en anglais, à laquelle s'ajoutent de nouveaux outils numériques, est un réel défi pédagogique qui permet cependant d'établir des stratégies d'enseignement susceptibles de favoriser l'apprentissage. Après avoir décrit les mécanismes permettant de mettre en place un programme évolutif de la L1 au M2, l'article présente plus spécifiquement l'évaluation d'une pédagogie innovante mise en place au niveau Master. Il met en lumière les réussites, les échecs et les limites de l'enseignement en géographie-aménagement anglophone tout en questionnant la place de l'enseignant-chercheur géographe et linguiste.

This article examines the implementation of an English taught geography course, which is associated with a specific pedagogy established for the Bachelor and Master levels, at the University of Cergy-Pontoise. It shows that the hybridization between disciplines in geography, planning, and English, supported by new digital tools, is a real pedagogical challenge. Nevertheless, this hybridization makes it possible to establish new teaching strategies that are likely to promote learning. After describing the mechanisms for setting up an evolving pedagogical program from $\mathrm{L} 1$ to $\mathrm{M} 2$, the article presents the assessment of an innovative pedagogy set up at the Masters level. It highlights the successes, failures, and limitations of teaching geography and planning in English. It also questions the roles of teachers and researchers as geographers and linguists.

\section{INDEX}

Mots-clés : pédagogie, géographie-aménagement, anglais, hybridation disciplinaire Keywords : Pedagogy, Geography and Planning, English, Disciplinary Hybridization

\section{AUTEUR}

\section{CLAIRE DOUSSARD}

Phd, Enseignante contractuelle, École Spéciale d'Architecture, chercheuse associée à l'UMR AUSser 3329 CNRS.

claire.doussard[at]gmail.com 